\title{
Spectral coding performance under free space optical medium
}

\author{
A.K Rahman ${ }^{1}$, C.B.M.Rashidi ${ }^{2}$, S.A Aljunid ${ }^{3}$, K.F. Tamrin ${ }^{4}$, Rudiyanto P. Jong ${ }^{5}$, D.N.A Zaidel ${ }^{6}$, \\ Y.S Samat ${ }^{7}$ \\ ${ }^{1,4,5,6,7}$ Faculty of Engineering, Department of Electrical Electronic, University Malaysia Sarawak, Malaysia \\ ${ }^{2,3}$ Advance Communication Engineering, Centre of Excellence, SCCE, University Malaysia Perlis, Malaysia
}

\section{Article Info \\ Article history: \\ Received Sep 28, 2018 \\ Revised Nov 26, 2018 \\ Accepted Dec 10, 2018}

\section{Keywords:}

APD noise

Conventional code

FSO communication

ZCC code

\begin{abstract}
This paper focus on performance of code Zero Correlation-Correlation (ZCC) in free space optical communication. The ZCC code has a superior characteristic which eliminate the overlapping code between any users. Due to this high class characteristic, the code improves the performance of the conventional code in free space optical environment. In this paper the analysis performance of bit error rate is considering the avalanche (APD) noise, thermal noise and multiuser interference. The result shows that ZCC code improve the performance of conventional code in term of number of users, power received and data bit rate.
\end{abstract}

Copyright (C) 2019 Institute of Advanced Engineering and Science. All rights reserved.

\section{Corresponding Author:}

Abdul Rahman Bin Kram,

Department of Electrical and Electronic Engineering,

University Malaysia Sarawak,

Fakulti Kejuruteraan, Jalan Dato Mohd. Musa, 94300 Kota Samarahan, Sarawak, Malaysia.

Email: karahman@unimas.my

\section{INTRODUCTION}

Free space optics (FSO) can describe as a sending very high bandwidth digital data from one point to another using a laser beam in a method similar to fiber optics, but directly through the atmosphere without the fiber. FSO also can be known as a concept of a narrow beam of light which is launched at a transmission station, transmitted through the atmosphere, and subsequently received at the receiving station [1], [6].

Free space optical communications have attracted a considerable attention for a variety of applications in the field of telecommunications. FSO applications span over a wide range from satellite links to robotics and generated interest from several distinct markets, namely: the last mile high bandwidth internet connectivity, the temporary high bandwidth data links, the mobile telephony back-haul (3G), satellite links as well as the various applications where the optical fibers cannot be deployed.

Recently, optical code-division multiple access (CDMA) schemes have been popular among researcher in designing the code which allows the multiple users to access the network simultaneously or asynchronously $[2,3,8,9,10]$. There are three types basic of division multiple access schemes. Firstly is time-division multiple access (TDMA) which divides access by time, secondly is frequency-division multiple access (FDMA) divides it by frequency and thirdly is code division multiple access (CDMA).

Among these three types of schemes CDMA is easier in channel assignment. There has been reported $[8,11,12,13,14]$ proposed of optical CDMA for free space optical communication and background light is the only consideration performance [4]. However for conventional code such as OOC code, the major weakness is the presence of overlapping weight in code or more well known as cross correlation. This condition will lead to existence of multiple access interference (MAI) noise [7, 15, 16, 17, 18, 19, 20]. 
Basically the MAI contribution is come from number of users. If large number of users involve the MAI as well will increase which lead to deterioration of the quality signal. Ultimately this condition can produce poor bit error rate (BER) where can drop below the acceptable standard communication BER (10-9).

Based on report Anuar[5], he have been develop the novel code which create zero cross correlation code with outstanding performance especially capable increasing large number of user with maintain under the acceptable BER. Therefore as one the key element in designing OCDMA is to reduce the cross correlation between code or user. In this paper we focus on ZCC code performance in free space optical communication channel. The comparison with conventional code that is OOC code in term of number of users, data bit rate and power received.

\section{RESEARCH METHOD}

A. Zero Cross Correlation Code

The number of users and length of the code for ZCC code can is given by [5]:

$$
K=w+1
$$

And

$$
L=w(w+1)
$$

where $K$ is the number of users, $L$ is the length of the code and $w$ is the weight of the code. The data bit rate is given by [4]:

$$
R_{b}=\frac{1}{T_{c} F}
$$

where $T c$ is the time interval

B. Probability of Error

The average number of absorbed photons at receiver can be denoted as:

$$
\lambda_{s} T_{c}
$$

where the photon absorption rate when ' 1 ' transmission occurs is given as:

$$
\lambda_{s}=\frac{\eta P_{r}}{h f_{0}}
$$

Meanwhile the chip interval time,

$$
T c=1 /(R b \times L)
$$

Where the $R b$ is the data bit rate of 1 bits/sequence-period, $P r$ is the total received laser power by the APD, $\eta$ is APD photodiode efficiency, $h$ is Plank's constant and $\mathrm{f} 0$ is the optical frequency. When laser transmit ' 1 ' or ' 0 ' the photon absorption rate can written as:

$$
\lambda=\left\{\begin{array}{l}
\lambda_{s}+\lambda_{b}+\frac{I_{b}}{e} \\
\frac{\lambda_{s}}{M_{e}}+\lambda_{b}+\frac{I_{b}}{e}
\end{array}\right.
$$

where $\lambda$ represent the total photon absorption rate due to signal background and APD bulk leakage current, $\lambda_{b}$ is the photon absorption rate due to the actual background light, $e$ is an electron charge, $\mathrm{I}_{\mathrm{b}} / \mathrm{e}$ is represent the contribution of the APD bulk leakage current to the APD output and $M e$ is the modulation extinction ratio of the laser output power in " 1 " or " 0 " state.

Spectral coding performance under free space optical medium (A.K Rahman) 
The general expression for bit error rate for FSO is given by:

$$
P e=P(0) \int_{-\infty}^{V t h} P(\text { error } / 0)+P(1) \int_{V t h}^{\infty}(\text { error } / 1)
$$

C. Orthogonal Optical Code (OOC)

Probability error can be express as

$$
\begin{aligned}
& P_{\text {ERROR }}=\frac{1}{2} P_{I_{I}}\left(P_{\text {FalseAlarm }}+P_{\text {Miss }}\right) \\
& P_{\text {ERROR }}=\frac{1}{2} \sum_{i=0}^{N-1} P_{I_{I}}\left[Q\left(\frac{T h-\mu_{b 0}}{\sigma_{b 0}^{2}}\right)+Q\left(\frac{\mu_{b 1}-T h}{\sigma_{b 1}^{2}}\right)\right]
\end{aligned}
$$

where the

$$
\begin{aligned}
& Q(\mathrm{x}) \text { is } \mathrm{Q} \text { function of BER, } \\
& \mathrm{x}=\log (\mathrm{BER}) .
\end{aligned}
$$

For unconditioned BER in presence of turbulent, we using the gamma-gamma distribution turbulence model combined with the conditioned BER which can be written as:

$$
P_{\text {ERROR }}=\left(\frac{2(\alpha \beta)^{\frac{\alpha+\beta}{2}}}{\Gamma(\alpha) \Gamma(\beta)} X^{\frac{\alpha+\beta}{2}} k_{\alpha-\beta}(2 \sqrt{\alpha \beta X})\right) \cdot\left[\frac{1}{2} \sum_{i=0}^{N-1} P_{I_{I}}\left(Q\left(\frac{T h-\mu_{b 0}}{\sigma_{b 0}^{2}}\right)+Q\left(\frac{\mu_{b 1}-T h}{\sigma_{b 1}^{2}}\right)\right]\right)
$$

D. Zero Cross Correlation Code (ZCC)

Probability of error can be express as

$$
\begin{aligned}
& P_{\text {ERROR }}=\frac{1}{2}\left(P_{\text {FalseAlarm }}+P_{\text {Miss }}\right) \\
& P_{\text {ERROR }}=\frac{1}{2} Q\left(\frac{T h-\mu_{b 0}}{\sigma_{b 0}^{2}}\right)+\frac{1}{2} Q\left(\frac{\mu_{b 1}-T h}{\sigma_{b 1}^{2}}\right)
\end{aligned}
$$

For BER in turbulent condition the BER will denote as:

$$
P_{E R R O R}=\left(\frac{2(\alpha \beta)^{\frac{\alpha+\beta}{2}}}{\Gamma(\alpha) \Gamma(\beta)} X^{\frac{\alpha+\beta}{2}} k_{\alpha-\beta}(2 \sqrt{\alpha \beta X})\right) \cdot\left(\frac{1}{2}\left(Q\left(\frac{T h-\mu_{b 0}}{\sigma_{b 0}^{2}}\right)+Q\left(\frac{\mu_{b 1}-T h}{\sigma_{b 1}^{2}}\right)\right)\right.
$$

\section{RESULTS AND ANALYSIS}

We present some numerical result using the following parameter: number of users $=40$, the wavelength of optical carrier is $825 \mathrm{~nm}$ and the bit rate data is $\mathrm{Rb}=156 \mathrm{Mbps}$. For $\mathrm{ZCC}$ code the $\mathrm{K}=4$ and $\mathrm{F}=160$ and for $\mathrm{OOC} \mathrm{K}=4$ and $\mathrm{F}=481$. The background light photon arrival rate $\lambda \mathrm{b}=1011$.

Figure 1 shows BER versus data bit rate between ZCC code and OOC code. The data bit rate is measured from $100 \mathrm{Mbps}$ to $3 \mathrm{Gbps}$. The number of users is set to 40 users and wavelength $\lambda$ is $825 \mathrm{~nm}$. Code length ZCC F is 160 and OOC F is 481 and both codes using code weight $K=4$. We can see that the ZCC code performs better compared to OOC code.

This is because the increasing of data bit rate will reduce the chip interval time (Tc) and sequenceperiod (F). This because the data bit rate is reverses proportional with chip interval time (Tc) and sequence- 
period $(\mathrm{F})$. Here due OOC code have a longer code length compare to ZCC it cause the data bit rate decrease. As we increase more bit rate the BER for OOC worse. This condition differs for ZCC which can support at a high data bit rate. At the standard bit error rate in communication which is 10-9 the OOC code can only survive approximately $400 \mathrm{Mbps}$ meanwhile for ZCC code it can reach approximately $1.7 \mathrm{Gbps}$.

Figure 2 shows the BER versus number of user. The number of user is measured from 5 to 160 users. The data bit rate is $156 \mathrm{Mbps}$ with code length $\mathrm{ZCC}$ is $\mathrm{F}=160$ and OOC code length is $\mathrm{F}=481$ and using same code weight $\mathrm{K}=4$. It shows that at BER $10-9$ the OOC code can support approximately 30 users but ZCC can reach approximately 80 users. For OOC code, the increasing number of users will increase as well the number probability of interference between users which can cause the increasing of bit error rate. This is because of it's own property OOC code which has the maximum cross-correlation equal to 1 . However for ZCC code the maximum cross-correlation is zero. In other word there is no overlapping code weight occurring in ZCC code. Therefore the bit error rate can be reduced.

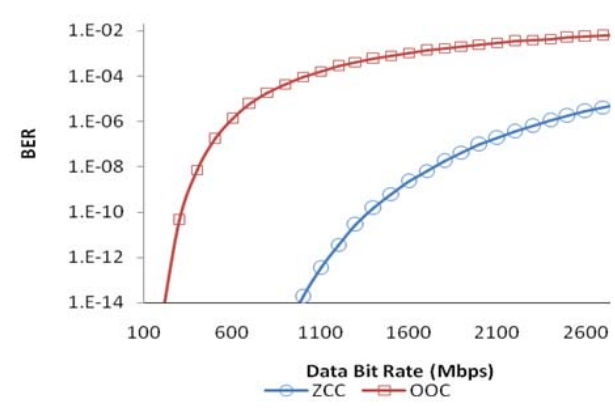

Figure 1. BER versus data bit rate(Mbps)

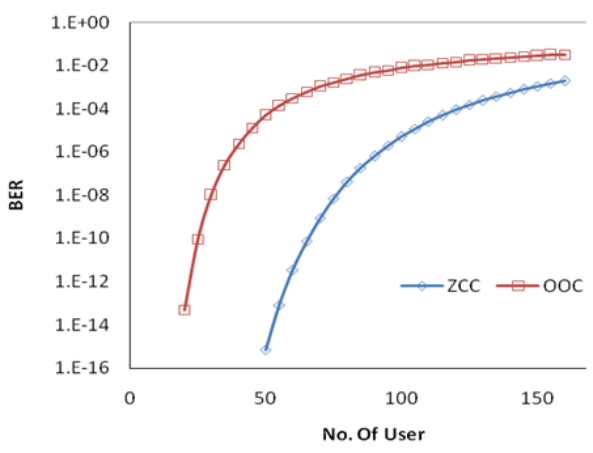

Figure 2. BER versus number of users

\section{CONCLUSION}

The performance of atmospheric OCDMA for zero cross correlation code with comparison OOC code has been analyzed. The ZCC code has been proven to superior performance in atmospheric condition compared to the OOC code. No overlapping between user give a big advantage for ZCC code which reduce BER factor. From numerical analysis ZCC can support high data bit rate up to gigabit per second with high number of users.

\section{ACKNOWLEDGEMENTS}

This work has been supported by MyRA grant Universiti Malaysia Sarawak.

\section{REFERENCES}

[1] J. Schuster, H. Willebrand, S. Bloom, E. Korevaar, Understanding the performance of Free Space Optics, in Journa pefrol of Optical Networking, 2003.

[2] J. Salehi, Code division multiple-access technique in optical fiber network - Part I: Fundamental principles, IEEE Trans. Commun., vol. 37, no. 8, pp. 824-833, Aug. 1989.

[3] J. Salehi and C. bracket, Code division multiple-access technique in optical fiber networks- Part II: System performance analysis,IEEE Trans. Commun., vol. 37, no.8, pp. 834-842, Aug. 1989.

[4] [4]R.M Gagliardi, Pulse-coded multiple-access in space optical communications, IEEE Journal on selected areas in communication, Vol.13, No.3, April, 1995.

[5] [5]Anuar. MS, S.A Aljunid, Minimizing correlation effect using zero cross correlation code in spectral amplitude coding optical code division multiple access, Optical Review Vol. 19, No. 1 (2012) 20-24.

[6] Willebrand, H.,Ghuman,B.S. Free-space Optics,Enabling Optical Connectivity in Today's Networks, Sams Publishing, Indianapolis. 2001.

[7] ]N. G. Tarhuni, T. O. Korhonen, M. Elmusrati, and E. Mutafungwa: Opt. Commun. 259 (2006) 655.

[8] S.A. Aljunid, M. D. A. Samad, M. Othman, M. H. Hisham, A.H. Kasiman and M. K. Abdullah, Development of Modified Double-Weight Code and its Implementation in Multi-Rate Transmissions. Networks, Communication, 2005 13th IEEE international Conference, Vol.1, ISSN 1531-2216, pp.5.

[9] Z.Wei et al., "Modified Quadratic Congruence Codes for Fiber Bragg Grating based Spectral Amplitude Coding Optical CDMA System”,Journal Lightwave Technology, Vol 19, Sept. 2001, 1274-1281. 
[10] X.Wang, "Keys towards practical OCDMA networks", 7th IEEE Int. Conf. on Optoelectronics, Fiber Optics and Photonics (Photonics2004) (Invited Paper), Dec. 2004.

[11] M.S. Anuar, S.A. Aljunid, N.M. Saad, S.M. Hamzah, New design of spectral amplitude coding in OCDMA with zero cross-correlation. Optics Communications (2009), Elsevier B.V.

[12] Guu-Chang Yang: Optical Orthogonal Codes with unequal auto- and cross-correlation constraints. IEEE Trans. on Information Theory, Vol.41, No.1, 1995, pp96- 106.

[13] Jawad. A. Salehi, F. R. K. Chung, and V. K. Wei: Optical orthogonal codes: Design, analysis, and applications. IEEE Trans. on Information theory, Vol.35, Nol.3, May 1989, pp595 - 605.

[14] W.C. Kwong and G.C. Yang, "Construction of $2 \mathrm{n}$ prime sequence codes for optical code division multiple access, IEE Proc. Commun, vol. 142, no. 3, pp. 141-150, 1995.

[15] M.S. Anuar, N.M. Saad, A. Mohammed, E.L. Balekir, "Development of a zero cross correlation code for spectral amplitude coding optical code division multiple access (OCDMA)," IJCSNS International Journal of Computer Science and Network Security, vol. 6, no. 12, Dec. 2006.

[16] S.A. Aljunid, M. Ismail, A.R. Ramli, B.M. Ali, M.K. Abdullah, "A new family of optical CDMA systems," IEEE Photonics Technology Letter, vol. 16, no. 10, Oct. 2004.

[17] Sang Wook Han "Optical CDMA with Optical Orthogonal Code" Multiuser Wireless Communication (EE381k) Class Project, Fall 2002.

[18] C. H. Lee: Fiber to the home using a PON infrastructure, J. Lightwave Technol., vol. 24, pp. 4568-4583, Dec.2006.

[19] J. S. Lee, Y. C. Chung, D. J. DiGiovanni: Spectrum-sliced fiber amplifier light source for multichannel WDM applications, IEEE Photon.Technol. Lett., vol. 5, pp. 1458-1461, Dec. 1993.

[20] M.S.Anuar, S.A. Aljunid, R.Badlishah, N.M.Saad, I.Andonovic "Performance Analysis of Optical Zero Cross Correlation in OCDMA System",Journal of Applied Sciences 7 (23):3819-3822, 2007, ISSN 1812-5654. 